Research Paper

\title{
Routine Pre-Treatment MRI for Breast Cancer in a Single-Payer Medical Center: Effects on Surgical Choices, Timing and Outcomes
}

\author{
Timothy J Vreeland ${ }^{1 凶}$, John S Berry IV ${ }^{2}$, Erika Schneble ${ }^{3}$, Doreen O Jackson³, Garth S Herbert ${ }^{3}$, Diane F \\ Hale$^{3}$, Jonathon M Martin³, Madeline Flores33, Adam R Pattyn', Kai Hata3, Guy T Clifton³, Aaron D \\ Kirkpatrick$^{5}$, George E. Peoples 6 \\ 1. Womack Army Medical Center. Department of Surgery. Fort Bragg, NC USA; \\ 2. Washington University School of Medicine. Department of Surgery. St. Louis, MO USA; \\ 3. San Antonio Military Medical Center. Department of Surgery. Ft Sam Houston, TX USA; \\ 4. Madigan Army Medical Center. Department of Surgery. Ft Lewis, WA USA; \\ 5. San Antonio Military Medical Center. Department of Radiology. Ft Sam Houston, TX USA; \\ 6. Cancer Vaccine Development Program. San Antonio, TX USA. \\ $\triangle$ Corresponding author: Timothy J Vreeland, MD, 2817 Reilly Rd, Fort Bragg, NC 28307 Cell phone: 6363465458 email: vreelant@gmail.com
}

(C) Ivyspring International Publisher. This is an open access article distributed under the terms of the Creative Commons Attribution (CC BY-NC) license (https://creativecommons.org/licenses/by-nc/4.0/). See http://ivyspring.com/terms for full terms and conditions.

Received: 2016.07.05; Accepted: 2017.03.08; Published: 2017.07.23

\begin{abstract}
Introduction: Pre-operative MRI is being used with increasing frequency to evaluate breast cancer patients, but the debate surrounding risks and benefits of this use continues. At our institution, we instituted a standardized protocol for pre-operative MRI. Here, we compare patients seen prior to routine use of MRI to those seen after and examine effects on surgical choices, timing and outcomes.

Methods: This is a retrospective review of a prospectively collected database of all new invasive breast cancers seen from January 2007 to December 2012. The control group (CG) did not receive MRI, while the MRI group (MRG) underwent MRI according to our pretreatment protocol. Groups were compared with regards to basic demographics, initial surgical choices, need for re-excision, and surgical timing. The electronic medical records of patients in the MRG who underwent mastectomy as their initial surgery were examined closely to determine the main factors leading to their choice of surgery. Finally, correlation between findings on MRI and final surgical pathology was analyzed.

Results: Of 282 patients included, 38 were in the CG and 244 in the MRG; the groups were well matched. The MRG had a significantly higher percentage of patients choosing initial mastectomy (MRG: $47.1 \%$ vs CG $21.1 \%, p=0.003$ ). Patients seen in the first 2 years of the study were less likely to choose mastectomy than those enrolled in the latter years $(29.2 \% \mathrm{vs} 48.6 \%, \mathrm{p}=0.004)$. The MRG had a lower chance of return to the operating room for re-excision $(15.2 \%$ vs $28.9 \%, p=0.035)$. The average time from initial imaging to initial surgery was approximately the same between groups (MRG: 39.7 days vs CG 42.1 days, $p=0.45$ ) and the MRG actually had shorter time to definitive (margin-negative) surgical management (MRG: 43.5 days vs CG: 50.3 days, $p=0.079$ ). One hundred-fifteen patients in the MRG underwent mastectomy as initial surgery. Of these, 64 (55.7\%) had no additional findings on MRI and chose mastectomy based on patient preference; 30 patients (26.1\%) (29 unilateral, 1 bilateral) had mastectomy because of MRI findings. Of the 31 breasts removed (29 unilateral and 1 bilateral mastectomies) because of MRI findings, 26 (83.9\%) had histologic findings that correlated with the MRI findings, while 5 (16.1\%) did not.

Conclusion: Patients receiving routine pre-treatment MRI had an increased mastectomy rate, but had a lower re-excision rate. We found no delay to initial surgical therapy and, perhaps more importantly, a slight decrease in time to margin-negative surgical therapy in the MRI group. Women choosing mastectomy after MRI did so because of personal preference over half of the time, while MRI findings influenced this choice in $26 \%$ of these women. When MRI findings did lead to mastectomy, these findings were confirmed by pathology results in the vast majority of cases.
\end{abstract}

Key words: Pre-Treatment Breast MRI, Breast Conserving therapy, Mastectomy, Re-excision Rate. 


\section{Introduction}

Over the past two decades, contrast enhanced MRI has been integrated into the clinical evaluation of breast cancer patients, and its use has increased significantly across the United States (1) and Canada (2). MRI is accepted as a screening tool in women who are at high risk of developing breast cancer based on increased sensitivity over mammography. Multiple professional societies (NCCN, ACS, ACR) now recommend screening MRI for women with a known genetic risk factor (BRCA, Cowden Syndrome, etc), certain environmental risk factors (radiation to chest between age 10 and 30), or an estimated lifetime risk of breast cancer of $20 \%$ (3-5). The indications for pre-treatment MRI in patients carrying a diagnosis of breast cancer, however, are much less clear. Current NCCN guidelines include a category 2B recommendation stating that MRI "may be used" for staging prior to operative intervention(6), while the American College of Radiology grades the use of MRI in intial workup of breast cancer as a 5 on a scale from 1 to 9 , classifying its use as "may be appropriate." (4) The lack of a clear consensus in the current literature on this issue contributes directly to the ambiguity of these recommendations.

The promise of MRI in the pre-treatment setting is the same as when used for screening, namely increased sensitivity. MRI sensitivity in detecting multicentric (MC) and contralateral disease has been quoted as high as $93 \%$ and $88 \%$ compared to $46 \%$ and $19 \%$, respectively, for conventional mammogram and ultrasound (7). This increased sensitivity seems to be particularly valid in patients with dense breasts (8) or with lobular cancers (9). In theory, this increase in sensitivity should lead to fewer positive margins, decreased re-excision rates, and potentially lower local recurrence rates if additional clinically relevant disease can be detected. The results of studies examining these issues, however, have been mixed.

While increased sensitivity can be a strength, it can also be a detriment if not coupled with appropriate specificity. MRI has been associated with delays in treatment, increased costs (in part due to workup of additional findings), and increased mastectomy rates. These delays and additional mastectomies may be justified if the MRI findings are confirmed by pathology findings and serve to reduce margin positivity, but this is not always the case. Bleicher et al. summarized these concerns well in their report. Compared to those without MRI, patients undergoing pretreatment MRI had a 22.4-day delay in pretreatment evaluation and an odds ratio for mastectomy of 1.80; simultaneously, there was no decrease in positive margins at lumpectomy, nor a decrease in conversion from breast conservation to mastectomy (10). Along the same lines, Aranout, et al. recently published a report of a population-based study of patients in Canada over a 10-year period in which they found that pre-treatment MRI was associated with increased post-diagnosis imaging and biopsies, more contralateral prophylactic mastectomies, and a greater than 30-day wait to surgery (2).

These two reports are representative of the literature available on this topic, with mainly retrospective studies that involve populations where MRI was used selectively and infrequently (only $14.8 \%$ of patients across the Aranout study had pre-treatment MRI), opening interpretation of any data on the subject to significant bias. Also, the inconsistent, non-standardized use of MRI fails to streamline the process, and could even adversely affect the diagnostic accuracy of MRI if interpretation is left to providers who are unfamiliar with its use. At our institution, we have unique advantages for evaluating routine use of pretreatment MRI. First, patients receive care as military beneficiaries, and pre-operative tests are performed without any need for insurance approval. Second, patients receive all of their cancer care in one facility, potentially streamlining their diagnostic work-up and transitions between providers. Finally, we instituted a diagnostic protocol in which all patients with biopsy-proven invasive breast cancer are scheduled for bilateral breast MRI upon pathologic diagnosis. Our single-payer, single institution system gives us a distinctive opportunity to study how efficiently a system can accomplish routine breast MRI and to examine the effects of this test on surgical outcomes. Barchie et al. reported our early experience with this protocol and showed that we had no delay in therapy. Furthermore, while mastectomies were increased, they found high MRI sensitivity and specificity for multifocal (MF)/MC disease $(89.5 \%$ and $84.2 \%$, respectively) (11). In this study, we examine our complete experience with standardized use of pre-treatment MRI over a 6-year period.

\section{Methods}

\section{Patient Selection}

This is a retrospective review of a prospectively collected database of all new invasive breast cancer cases seen at San Antonio Military Medical Center (SAMMC) from January 2007 to December 2012. We prospectively gathered demographic, diagnostic, 
pathologic and treatment information for all patients seen in our Breast Cancer Comprehensive Care Conference (BC4) clinic during this time. In September 2007, we instituted a protocol of standardized preoperative bilateral breast MRI for all biopsy-proven invasive cancers. The protocol was approved and monitored by the SAMMC Institutional Review Board. Patients who did not receive MRI, most of whom were evaluated from January 2007 to September of 2007, constituted a control group (CG), while patients seen after this date who received MRI were considered the MRI group (MRG). The SAMMC IRB waived the requirement for informed consent prior to MRI. Patients were excluded if they were treated with neo-adjuvant therapy, refused surgery, had metastatic disease, had incomplete data, or were lost to follow-up (Figure 1, consort diagram).

\section{Treatment Protocol}

The pretreatment MRI protocol was administered exclusively at SAMMC to Tricare beneficiaries. All patients with mammographic abnormalities underwent image-guided biopsy. Patients with biopsy proven invasive carcinoma were placed into the institutionally supported imaging pathway. A renal function panel was obtained and the patient was scheduled for MRI at their earliest convenience. On occasion, patients with BIRADS 5 lesions underwent MRI prior to biopsy at the discretion of the attending radiologist. The patient then underwent MRI, with the goal of finalized interpretation prior to the patient's appointment in the multi-disciplinary BC4 clinic. Here, each patient was seen by a surgeon, a radiation oncologist and a medical oncologist. Providers then met, generally that same afternoon, in a multi-disciplinary conference, which included providers from General Surgery/Surgical Oncology, Radiation Oncology, Diagnostic Radiology, Medical Oncology, Pathology, as well as a Breast Care Nurse.

\section{MRI Technique}

MRI was conducted with 1.5 Tesla Marconi and Siemens Esprit magnets with InVivo dedicated seven channel breast coils. Core sequences include axial STIR, 3D axial T1, T2 sagittal fat saturated, pre and post dynamic contrast axial T1 SPGR fat saturated images with and without subtraction. Post-contrast images were repeated to $5 \mathrm{~min}$ with $60 \mathrm{sec}$ temporal resolution. Slice thickness was $0.9 \mathrm{~mm}$ with $0.89 \mathrm{~mm}$ in-plane resolution. Matrix size was $381 \times 448$ with field of view of $34 \times 30 \mathrm{~cm}$. Coronal and sagittal reformats were obtained. All studies were performed at SAMMC and interpreted by board certified staff radiologists. The MRI was scheduled at the earliest possible time, regardless of menstrual cycle, to avoid delay of definitive management, accepting possible negative impact on sensitivity and specificity (12). Contrast administration was automated with a $0.1 \mathrm{mmol} / \mathrm{kg}$ gadolinium injection followed by a $10 \mathrm{~mL}$ flush.

\section{Data Collection and Analysis}

The breast cancer database was reviewed for basic demographic data, histological diagnosis, surgical interventions, and temporal relationships between imaging, biopsy and surgery dates. Clinical stage was determined by initial surgical specimen. Time from initial radiologic detection to final (margin-negative) surgical management was recorded in order to analyze any delay in surgical treatment. The records of patients undergoing mastectomy after MRI were reviewed very closely to determine the reason for their choice of mastectomy. Pre-operative clinic notes were examined for the discussion between patient and surgeon/oncologist and, when possible, a reason for mastectomy was assigned based on these notes. Final pathology reports from these cases were also closely examined for correlation with imaging findings.

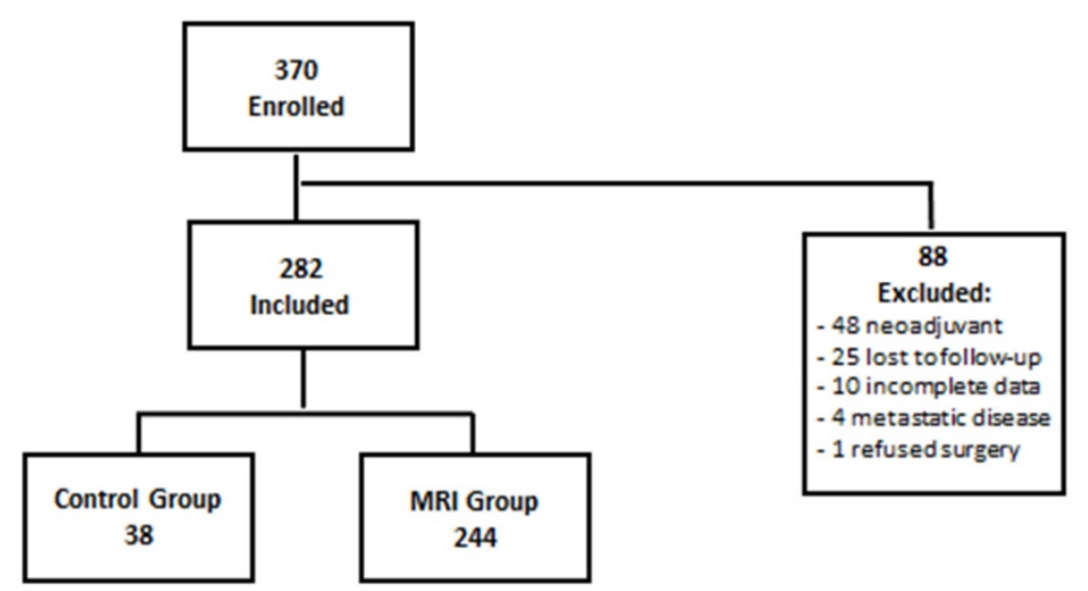

Figure 1. Consort Diagram 
Fisher's exact test was used to investigate if proportional differences existed between the MRG and the CG with regards to histological subtype, stage, mastectomy rate, re-excision rate, and rate of conversion from BCT to mastectomy. The Student $t$ test was used for statistical analysis of continuous variables. Mann-Whitney $U$ test was used for comparison of independent, nonparametric variables, including age, tumor size, and time from diagnosis to surgery.

\section{Results}

\section{Patients}

A total of 370 patients with invasive breast cancer were seen in BC4 clinic between January 2007 and December 2012. Of those patients, 88 patients were excluded: 48 underwent neoadjuant therapy, 25 were lost to follow-up, 10 had incomplete data available, 4 had metastatic disease and 1 refused surgery (Figure 1). Of the remaining 282 patients, 38 $(13.5 \%)$ did not receive a pre-treatment MRI, most being seen immediately prior to institution of the MRI protocol, and made up the control group (CG). The other 244 patients $(86.5 \%)$ received pre-treatment MRI per protocol and, thus, were the MRI group (MRG). The MRG and CG were very well matched with respect to age, tumor size, histology, tumor grade, nodal status, ER positivity, HER2 positivity, and triple negative disease $(p>0.05)$ (Table 1$)$.

Table 1. Demographics

\begin{tabular}{llll}
\hline & MRI & CG & p value \\
\hline Age & 58.60 & 59.10 & 0.78 \\
Size $(\mathrm{cm})$ & 1.79 & 1.56 & 0.34 \\
Ductal Histology & $85.0 \%$ & $86.5 \%$ & 0.81 \\
High Grade & $20.0 \%$ & $16.2 \%$ & 0.59 \\
Node Pos & $30.0 \%$ & $28.6 \%$ & 0.86 \\
ER- & $14.6 \%$ & $18.2 \%$ & 0.59 \\
HER2+ & $12.8 \%$ & $3.0 \%$ & 0.10 \\
Triple Neg & $9.4 \%$ & $15.2 \%$ & 0.30 \\
\hline
\end{tabular}

\section{Initial Surgical Procedure}

Overall, 159 (56.4\%) patients chose BCT, while $123(43.6 \%)$ patients chose mastectomy as initial procedure. The MRG had a significantly higher percentage of patients choosing mastectomy as their initial procedure compared to the CG $(47.1 \%$ vs $21.1 \%$, $\mathrm{p}=0.003$ ). The rate of mastectomy as initial procedure changed over time. Regardless of group, patients seen in the first 2 years of the study $(2007-2008, n=72)$ were less likely to choose mastectomy than those enrolled in the latter years $(2009-2012, \mathrm{n}=209)(29.2 \%$ vs $48.6 \%$, $p=0.004$ ). Limiting analysis to the MRG, patients prior to 2009 were also less likely to choose mastectomy compared to those seen in 2009 and afterwards, thought this difference lost significance $(36.6 \%$ vs $49.3 \%, \mathrm{p}=0.14$ ).

Within the MRG, mastectomy patients had more advanced and high-risk disease than those choosing BCT. While MRG mastectomy and BCT patients were similar in age, grade, ER status, and percentage with triple negative disease, MRG mastectomy patients had significantly larger tumors $(p<0.01)$, more HER2+ disease $(p=0.01)$, and more node positive disease $(\mathrm{p}=0.05)$ (Table 2$)$.

Table 2. MRG Demographics

\begin{tabular}{llll}
\hline & BCT & Mastectomy & p value \\
\hline Age & 59.6 & 57.7 & 0.20 \\
Size & $\mathbf{1 . 4 4}$ & $\mathbf{2 . 1 5}$ & $<\mathbf{0 . 0 1}$ \\
Ductal Histology & $\mathbf{9 0 . 2} \%$ & $\mathbf{7 9 . 5 \%}$ & $\mathbf{0 . 0 2}$ \\
High Grade & $17.9 \%$ & $22.2 \%$ & 0.40 \\
Node Pos & $24.4 \%$ & $35.9 \%$ & 0.05 \\
ER- & $13.0 \%$ & $16.2 \%$ & 0.48 \\
HER2+ & $\mathbf{7 . 5} \%$ & $\mathbf{1 8 . 4} \%$ & $\mathbf{0 . 0 1}$ \\
Triple Neg & $9.1 \%$ & $9.6 \%$ & 0.88 \\
\hline
\end{tabular}

\section{Re-excision Rate}

Though there was a clear increase in mastectomy as initial surgical choice within the MRG, this group also had a lower chance of return to the operating room for re-excision $(15.2 \%$ vs $28.9 \%, p=0.035)$. Limiting analysis to patients who initially chose BCT, the MRG still had a lower rate of re-excision, though not significantly ( 28.7 vs $36.7 \%$, $\mathrm{p}=0.39$ ).

\section{Surgical Timing}

We examined the average time for each group to get from initial imaging with suspicious findings to surgical management. From imaging to initial surgery, the two groups averaged roughly the same interval (MRG: 39.7 days vs CG 42.1 days, $p=0.45$ ). We next examined surgical timing of the two groups in reference to a potentially more meaningful time point, time from initial imaging to definitive (margin-negative) surgical management. The MRG actually reached this endpoint on average one week faster than the CG, with a trend toward statistical significance (MRG: 43.5 days vs CG: 50.3 days, $\mathrm{p}=0.079$ ).

\section{MRI Patients Choosing Mastectomy}

There were 115 patients in the MRG who underwent mastectomy as initial surgery; these patients were closely reviewed to determine the reason for mastectomy. The results of this analysis are demonstrated in Figure 2. The reason for mastectomy could not be clearly determined in $3(2.6 \%)$ patients. Seventeen $(14.8 \%)$ patients had a contraindication to 


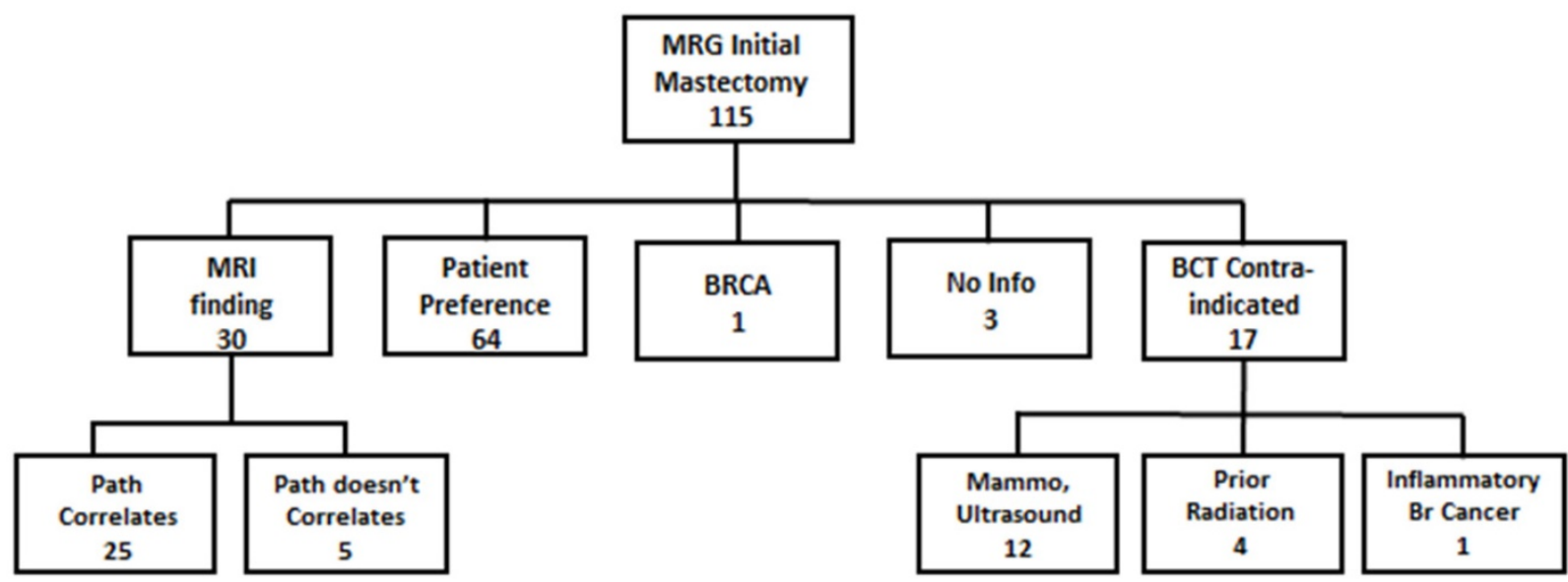

Figure 2. MRG Patients chosing mastectomy

BCT prior to MRI (12 with MC disease on mammogram or ultrasound, 4 with a history of prior radiation, and 1 with inflammatory breast cancer), so their decision was not affected by MRI results. One patient was found to be BRCA positive prior to surgery and elected to undergo bilateral mastectomy as a result. Thirty patients $(26.1 \%)$ had mastectomy because of an MRI finding (1 patient with tumor $>5 \mathrm{~cm}$, 1 with chest wall involvement, 4 with MF disease and 24 with MC disease). Sixty-four (55.7\%) patients had no additional findings on MRI and instead had mastectomy based on patient preference.

\section{Bilateral Mastectomy}

A total of twelve patients in the MRG had bilateral mastectomies, 2 because of imaging findings and 10 for prophylaxis. Of the 2 patients with imaging findings, 1 patient had findings of bilateral disease on mammogram (MRI did showed a corresponding finding), with corresponding pathology results. The other patient had no mammographic evidence of disease on the contralateral side, but MRI showed an area of suspicion, which was recommended for biopsy. This patient chose to forego second biopsy and instead to have bilateral mastectomies. Final pathology from the contralateral breast did show DCIS in the area that was suspicious on MRI. In the remaining 10 patients, contralateral MRI was negative and pathology showed no areas of DCIS or invasive malignancy. Overall, MRI correctly identified two cases of contralateral disease, one of which was not seen on other imaging modalities.

\section{MRI/Pathology Correlation}

Of the 31 breasts removed from 30 patients (29 unilateral, 1 bilateral mastectomies) because of new MRI findings, 26 (83.9\%) had histologic findings that correlated with the MRI results. Conversely, 5 (16.1\%) had no histologic correlate of MRI findings, 4 of which were thought to have MC disease and one thought to have MF disease. Overall, of the 244 patients who underwent MRI, 30 (12.3\%) underwent mastectomy because of a positive MRI; 5 patients with false positive results and 25 with true positive results. All 5 patients with false positive MRI findings were offered MRI guided biopsy of their additional MRI finding, but refused biopsy and instead chose to move forward with mastectomy.

\section{Discussion}

In our retrospective review of this prospectively collected data set, we found that after a standardized protocol of pre-operative MRI was initiated, patients who underwent MRI had a higher mastectomy rate than historical controls without MRI, but had less need for re-excision. Routine pretreatment MRI was not associated with delay in care, with initial surgery occurring in approximately the same interval after diagnosis, and margin-negative surgery actually being accomplished more quickly in patients receiving MRI. Importantly, over half of the patients in the MRG choosing mastectomy did so because of their preference, not because of the MRI findings. Additionally, roughly $10 \%$ of patient had true positive MRIs leading to mastectomy, while only 5 patients (2.1\%) underwent mastectomy because of false positive findings on MRI.

A number of studies have reported increased mastectomy rate when women undergo pre-operative MRI, and our results reinforce this finding. While there is clearly a correlation between MRI and mastectomy, a direct causal relationship is not as clear. Our study is based on retrospective analysis and a comparison of historical controls to more recently evaluated patients. This difference in temporal evaluation of patients makes the observed difference difficult to interpret as mastectomy rates across the United States have been increasing over the last 10 
years. A recent report of NSQIP data from 2005-2011 showed a relative increase in mastectomy of $2.9 \%$ per year during that time (13). Additionally, the SEER database showed the mastectomy rate in 2011 was $46 \%$ (14), which is roughly the same as the mastectomy rate in the MRG. Meanwhile, our control group mastectomy rate of $17.9 \%$ was quite low compared to the national average at that time (roughly 40\%) (13). Furthermore, even within our own study, there was an increase in the mastectomy rate comparing patients from the first 2 years to later patients. This difference may be in part due to the MRG all being treated later in the trial, but even within the MRG, an increase in mastectomy in the latter portion of the trial was observed, though this difference was not as large.

The relationship between MRI and mastectomy is indeed complicated, as the social acceptance of mastectomy has evolved during the same time period in which MRI use has increased, making it difficult to identify the causative factor. Aranaout et al. found an increase in mastectomy associated with MRI use, but the use of MRI increased from 3\% at the beginning of their study (2003) to $24 \%$ at the end (2012), during roughly the same time that Lucas, et al. showed an increase in mastectomy rates independent of any specific imaging $(2,13)$. Given the complicated nature of literature on this issue, we closely reviewed charts of MRG patients choosing mastectomy to discern the reasons for this choice. We found that over half of the patients choosing mastectomy after MRI did so because of choice, despite having negative MRIs. Only $26 \%$ of women choosing mastectomy in the MRG did so because of MRI findings. Indeed, the increase in MRI use over the past decade has coincided with, but did not necessarily cause, the trend of increased mastectomy during the same time. Ultimately, the use MRI in our study, similar to multiple others, is correlated with increased mastectomy, but was not the only cause of this increase.

While a certain percent of mastectomies may be explained by population trends, we were able to identify a population of patients whose decision was directly attributed to MRI findings. As noted above, the decision to undergo initial mastectomy was affected by MRI findings in roughly $12.3 \%$ of patients undergoing MRI. Of these patients, pathology results confirmed that the vast majority (83.9\%) of these findings correlated with DCIS or invasive cancer, which likely would have likely necessitated a second trip to the operating room for re-excision. This result confirms the early findings reported by Barchie et al. on the use of routine pretreatment MRI, which showed a high specificity for MC and MF disease (11). Additionally, we did identified a decreased re-excision rate in the MRG, implying that, for some, the increased sensitivity of MRI did have clinical benefit and, potentially, some of the mastectomies in the MRG may have been justified. What we cannot determine from our data set is whether these additional findings would have affected long-term oncologic outcomes. Randomized, prospective data are needed to determine the ultimate meaning of this increase in mastectomy that we, and others, have noted. Importantly, the five patients with false positive MRIs were offered MRI-guided biopsy, but refused. In order to avoid similar unnecessary mastectomies, patients who do undergo pre-treatment MRI should be counseled regarding the known possibility of false-positive findings and the need for biopsy of additional findings.

The other major criticism of pretreatment MRI has been delays in definitive surgical management. As noted earlier, Bleicher et al. found a 22.4-day delay in pretreatment evaluation for patients receiving MRI (10). These delays are attributed to the process of carrying out the test (need for approval, the time needed to schedule and actually complete the test), as well as the additional findings of MRI (the time needed to work up any new findings with additional imaging and/or biopsies). In our trial, however, we found no increase in time to surgery for the MRG. This finding, which is in stark contrast to most contemporary data, is likely in part attributed to ubiquitous and protocolized use of "in-house" MRI, which streamlines the process. Additionally, the ability to complete the MRI and any further work-up within a single institution decreases delays associated with scheduling and completing tests at multiple facilities. While this may not be applicable to all health systems, our data show that it is possible to obtain pre-operative MRI without significant delay.

In addition to time to initial surgery, we examined a potentially more clinically meaningful end-point, time to margin-negative resection. Likely due to a decreased re-excision rate in the MRG, we found that pretreatment MRI actually led to a trend towards decreased time to margin-negative resection. Ultimately, rather than basing merit of a pre-operative test on how quickly a patient undergoes an initial operation, a better metric might be time to completion of surgical treatment (to include reoperation for positive margins). This outcome better correlates with the start of recovery from surgery, start of adjuvant therapies, and, importantly, return to regular life without the stress of waiting for the result of another pathologic assessment. We suggest that time to margin-negative surgery, rather than time to initial surgery, is a more meaningful end-point for the evaluation of a pre-operative imaging modality. 
While our results are interesting, our institution is somewhat unique. We have a single, large medical center where the majority of our patients receive all of their care, to include all imaging and image-guided biopsies. Also, almost all of our patients fall within a single payer system, with no debate regarding insurance coverage of diagnostic tests that are not currently standard-of-care. Perhaps most importantly, we have instituted a standardized protocol of pre-operative MRI. Our results indicate that these factors allow for the completion of pre-operative MRI without any delay in care. While this may not translate to every health care system, it offers a glimpse of what is possible and indicates that if such a system can be instituted, concern for delaying a patient's care should not dissuade clinicians from the use of MRI.

There are several limitations to this study. First, this is a retrospective review with a non-randomized and non-contemporary control group. This introduces a number of issues, particularly because the rate of mastectomy has increased nationwide during the time of our study. Additionally, our control group is rather small, opening it to additional bias, such as the low number of women choosing mastectomy in the CG, as discussed above. Finally, our patient register is not set up to maintain lengthy follow-up records and, thus, we do not have long-term outcomes data on our patients. One of the true tests for pre-treatment MRI will be whether the increased sensitivity and change in surgical approach will affect oncologic outcomes. Specifically, will a decrease in positive margins with pre-operative MRI lead to a decrease in the local recurrence rate and, potentially, a decrease in disease-specific mortality? Unfortunately, our data does not allow us to comment on this potential outcome.

\section{Conclusion}

In conclusion, we have performed a retrospective review of our prospectively gathered database of breast cancer patients at our institution. We instituted a protocol to accomplish routine pre-treatment MRI for all women with a diagnosis of invasive breast cancer and compared patients who underwent pretreatment MRI to those who did not. Patients receiving MRI did have increased mastectomy rates as expected from previous reports, but had a lower re-excision rate. We found no delay to initial surgical therapy and perhaps more importantly, a slight decrease in time to margin-negative surgical therapy in the MRG. Women choosing mastectomy after MRI did so because of personal preference (and despite negative MRI findings) over half of the time, while MRI findings influenced this choice in $26 \%$ of these women. When MRI findings did lead to mastectomy, these findings were confirmed on histology in the vast majority of cases. Future studies will be focused on applying this protocol in non-invasive disease and comparing our invasive patients to those of other similar military hospitals, where pre-treatment MRI is used more sparingly to assess oncologic outcomes.

\section{Competing Interests}

The authors have declared that no competing interest exists.

\section{References}

1. Stout NK, Nekhlyudov L, Li L, Malin ES, Ross-Degnan D, Buist DS, et al. Rapid increase in breast magnetic resonance imaging use: trends from 2000 to 2011. JAMA internal medicine. 2014;174(1):114-21.

2. Arnaout A, Catley C, Booth CM, McInnes M, Graham I, Kumar V, et al. Use of Preoperative Magnetic Resonance Imaging for Breast Cancer: A Canadian Population-Based Study. JAMA Oncol. 2015;1(9):1238-50.

3. [Internet] NCCN. Breast Cancer Screening and Diagnosis. https://www.nccn. org/professionals/physician_gls/pdf/breast-screening.pdf

4. Mainiero MB, Lourenco A, Mahoney MC, Newell MS, Bailey L, Barke LD, et al. ACR Appropriateness Criteria Breast Cancer Screening. J Am Coll Radiol. 2013;10(1):11-4.

5. Saslow D, Boetes C, Burke W, Harms S, Leach MO, Lehman CD, et al. American Cancer Society guidelines for breast screening with MRI as an adjunct to mammography. CA Cancer J Clin. 2007;57(2):75-89.

6. [Internet] NCCN. Breast Cancer. http://www.nccn.org/professionals/ physician_gls/pdf/breast.pdf.

7. Hollingsworth AB, Stough RG, O'Dell CA, Brekke CE. Breast magnetic resonance imaging for preoperative locoregional staging. American journal of surgery. 2008;196(3):389-97.

8. Sardanelli F, Giuseppetti GM, Panizza P, Bazzocchi M, Fausto A, Simonetti G, et al. Sensitivity of MRI versus mammography for detecting foci of multifocal, multicentric breast cancer in Fatty and dense breasts using the whole-breast pathologic examination as a gold standard. AJR Am J Roentgenol. 2004;183(4):1149-57.

9. Bedrosian I, Mick R, Orel SG, Schnall M, Reynolds C, Spitz FR, et al. Changes in the surgical management of patients with breast carcinoma based on preoperative magnetic resonance imaging. Cancer. 2003;98(3):468-73.

10. Bleicher RJ, Ciocca RM, Egleston BL, Sesa L, Evers K, Sigurdson ER, et al. Association of routine pretreatment magnetic resonance imaging with time to surgery, mastectomy rate, and margin status. J Am Coll Surg. 2009;209(2):180-7; quiz 294-5.

11. Barchie MF, Clive KS, Tyler JA, Sutcliffe JB, Kirkpatrick AD, Bell LM, et al. Standardized pretreatment breast MRI--accuracy and influence on mastectomy decisions. J Surg Oncol. 2011;104(7):741-5.

12. Liberman, L. Breast MRI: Diagnosis and Intervention. New York: Springer Science and Business Media; 2005.

13. Lucas DJ, Sabino J, Shriver CD, Pawlik TM, Singh DP, Vertrees AE. Doing more: trends in breast cancer surgery, 2005 to 2011. Am Surg. 2015;81(1):74-80.

14. Lizarraga I, Schroeder MC, Weigel RJ, Thomas A. Surgical Management of Breast Cancer in 2010-2011 SEER Registries by Hormone and HER2 Receptor Status. Ann Surg Oncol. 2015;22 (Suppl 3):S566-72. 